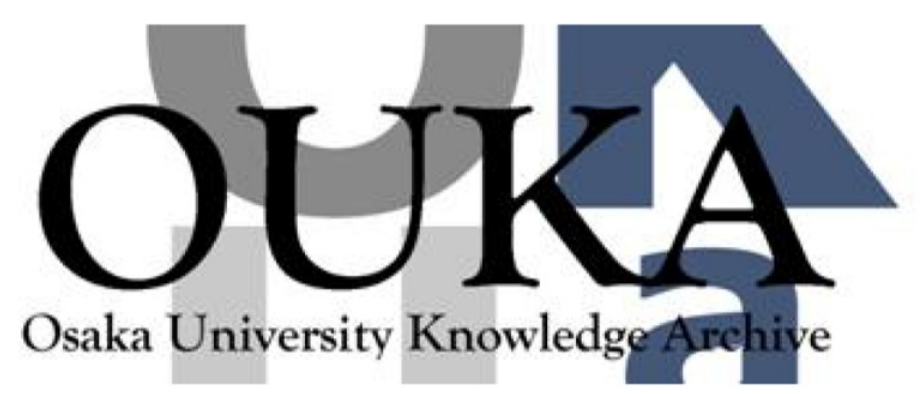

\begin{tabular}{|c|c|}
\hline Title & $\begin{array}{l}\text { Carrier generation and transport in discotic } \\
\text { liquid crystal studied by time of flight method }\end{array}$ \\
\hline Author(s) & Nakayama, H.; Ozaki, M. ; Schmidt, F. W. et al. \\
\hline Citation & $\begin{array}{l}\text { IEEE International Conference on Conduction and } \\
\text { Breakdown in Dielectric Liquids, ICDL. p. } 622- \\
\text { p. } 625\end{array}$ \\
\hline Issue Date & 1999-07 \\
\hline oaire:version & VoR \\
\hline URL & https://hdl. handle. net/11094/14086 \\
\hline rights & $\begin{array}{l}\text { c1999 IEEE. Personal use of this material is } \\
\text { permitted. However, permission to } \\
\text { reprint/republish this material for advertising } \\
\text { or promotional purposes or for creating new } \\
\text { collective works for resale or redistribution } \\
\text { to servers or lists, or to reuse any } \\
\text { copyrighted component of this work in other } \\
\text { works must be obtained from the IEEE. }\end{array}$ \\
\hline Note & \\
\hline
\end{tabular}

Osaka University Knowledge Archive : OUKA

https://ir. Library. osaka-u. ac. jp/

Osaka University 


\title{
Carrier Generation and Transport in Discotic Liquid Crystal Studied by Time of Flight Method
}

\author{
H. Nakayama ${ }^{1}$, M. Ozaki ${ }^{1}$, W. F. Schmidt ${ }^{1,2}$ and K. Yoshino ${ }^{1}$ \\ ${ }^{\prime}$ Department of Electronic Engineering, Graduate School of Engineering, Osaka University, \\ 2-1 Yamada-Oka, Suita, Osaka 565-0871, Japan \\ ${ }^{2}$ Department of Physics, Berlin Free University, Dahlemer Weg 172A, 14167 Berlin, Germany
}

\begin{abstract}
Carrier mobility in discotic liquid crystal, 2, $3,6,7,10,11$-hexahexyloxytriphenylene (HHOTP) has been evaluated by the analysis of a time-of-flight signal by taking a spatial distribution of generated charge carrier density into consideration. Photo-carrier generation process in HHOTP has been discussed in terms of Onsager model.
\end{abstract}

\section{Introduction}

In organic liquids, free electrons with large mobility depending on the molecular structure are confirmed to exist [1, 2]. Direct observation of holes in dielectric liquids, however, is highly limited so far [3]. On the other hand, only ionic carrier mobility, which is related with the viscosity of the fluid by Walden's rule, has been reported in ordinary liquid crystals such as nematic, smectic and cholesteric liquid crystals $[4,5]$. However, recently, observations of hole mobility have been reported in discotic liquid crystals such as $2,3,6,7,10,11$. hexahexylocytriphenylene (HHOTP) in which discshaped aromatic molecular core stack in one direction and form a columnar structure $[6,7]$.

In this paper, we discuss the time-of-flight signal in the discotic liquid crystal HHOTP by taking the spatial distribution of initial charge carrier into consideration and the carrier generation process by light irradiation in terms of Onsager model.

\section{Experimental}

HHOTP, whose molecular structure is shown in Fig. 1 , was synthesized and purified by column chromatography utilizing silica gel and chloroform/benzene mixture $(4 / 1)$ as eluent, and then recrystallized from acetone [8]. The discotic phase of HHOTP was confirmed in the temperature range between $53^{\circ} \mathrm{C}$ and $96^{\circ} \mathrm{C}$, which coincides with the reference [9].

The HHOTP sample was introduced in a sandwich cell composed of two parallel ITO (indium-tin-oxide)coated quartz plates by capillary effect. The polyethylene-terephthalate (PET) sheets of various thickness were used as spacers. By cooling the sample from the isotropic phase, a columnar structure of molecular alignment perpendicularly to ITO plates was realized in the discotic phase, which was confirmed by optical microscopic observation.

Carrier mobility was evaluated by a time-of-flight method[10] utilizing the third harmonics generation (THG) of Q-switched Nd:YAG light (355nm) of $20 \mathrm{~ns}$ in pulse width as an exciting light source. The photon flux was measured utilizing a portable power meter (Gentec SERIES3130).

\section{Results and Discussion}

Photoinduced current signal in HHOTP was strongly dependent on the polarity of the electrode through which

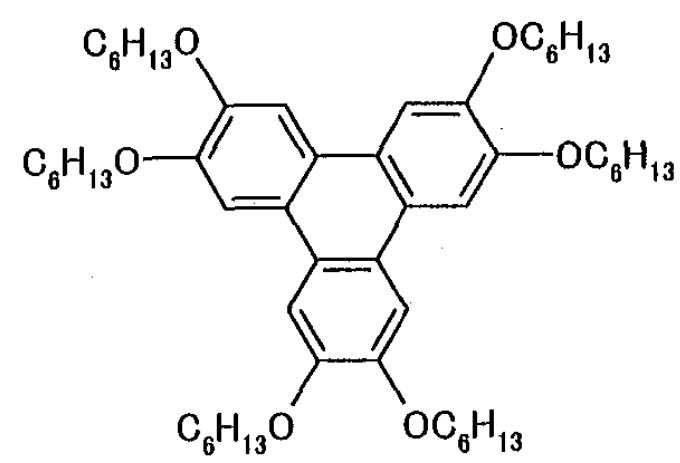

Fig. 1. Molecular structure of the discotic liquid crystal HHOTP, used in this study. 


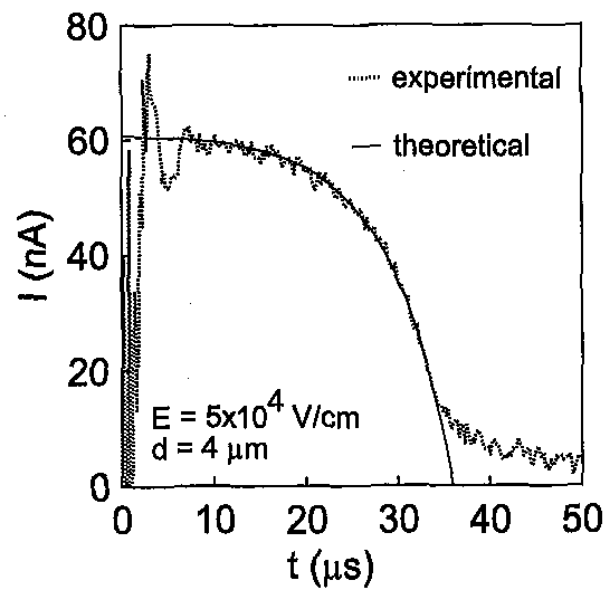

Fig. 2. Time response of photo-induced current signal to the pulsed laser light in the liquid crystalline phase of HHOTP for the positive charge carrier. A theoretical fitting curve is shown: by a solid line.

\section{the light impinges on the HHOTP sample.}

Figure 2 shows photoinduced current signal in the discotic liquid crystal phase for the positive bias in which the applied voltage of the irradiated side electrode is positive in polarity. In the case of negative polarity of irradiating side electrode, the response was much slower.

It should be noted in Fig. 2 that a clear break point corresponding to the carrier transit between electrodes was not clearly observed in time-of-flight method but slightly smeared out. In such a case, the results may be interpreted in terms of either short life time of photoinjected carrier or dispersed type of carrier migration of non-Gaussian packed form. Even in such a case, double logarithmic plots of induced current signal sometimes gives a knee, from which the transit time of carrier front between electrodes is evaluated.

In this study, however, we interpreted the results in terms of distribution of carrier generation area due to relatively small absorption coefficient of sample at the wavelength of the exciting light source. That is, we demonstrated that even for the Gaussian type carrier migration with long enough life time, clear kink becomes to be obscure in the time-of-flight signal. However, from such signal, mobility can be evaluated.

In such a case, the distribution of charge carriers density $\sigma$ in the cell is given by eq. (1) as the function of distance $(x)$ from the electrode through which the light pulse penetrates,

$$
\sigma(x)=\sigma_{0} e^{-\alpha x}
$$

where $\alpha$ is the absorption coefficient and $\sigma_{0}$ is the carrier density formed at the electrode interface.

When the distribution of charge carriers density in the cell is expressed by eq. (1), the total charge $Q(t)$ which drifts between electrodes at time $t$ is given as the integration of the charge carriers density of eq. (1).

$$
Q(t)=\int_{0}^{x_{t}} \sigma_{0} e^{-\alpha x} d x=\frac{1}{\alpha} \cdot \sigma_{0}\left(1-e^{-\alpha d\left(1-t / t_{\mathfrak{\tau}}\right)}\right),
$$

where $d$ is the thickness of the cell, $t_{\tau}$ is the transit time of carrier. Therefore expected current signal $l(t)$ at time $t$ is given as follows

$$
\begin{aligned}
I(t) & =Q(t) \cdot \frac{v}{d} \\
& =\frac{1}{t_{\tau}} \cdot \frac{Q(0)}{\left(1-e^{-\alpha d}\right)} \cdot\left(1-e^{-\alpha d\left(1-t / t_{\tau}\right)}\right),
\end{aligned}
$$

when $v$ is the drift velocity of the carrier.

The mobility measurement was performed in the range between room temperature and $110^{\circ} \mathrm{C}$, which covered all phases indicated in HHOTP. A theoretical fitting curve to the experimental trace with $t_{\tau}$ of $30 \mu$ s is shown by a solid line in Fig. 2. It should be noted that this is the case of a positive bias. Therefore, the signal is due to the migration of positive carrier. Deviation of the experimental signal in the tail part from the theoretical curve should be due to the migration of carriers, which are released

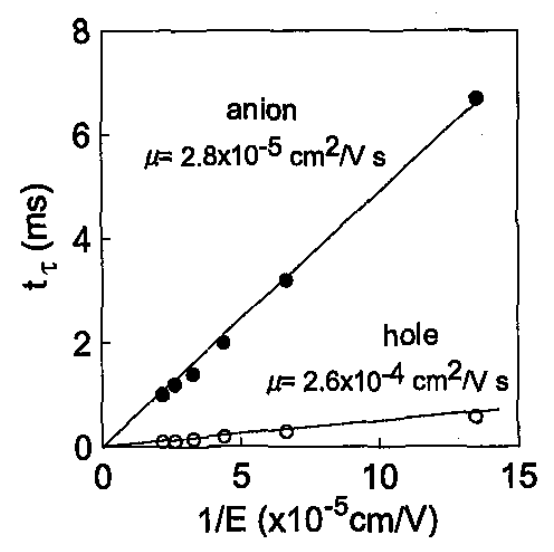

Fig. 3. Field strength dependences of transit time for positive (open circles) and negative (closed circles) carriers in HHOTP in the discotic phase. 


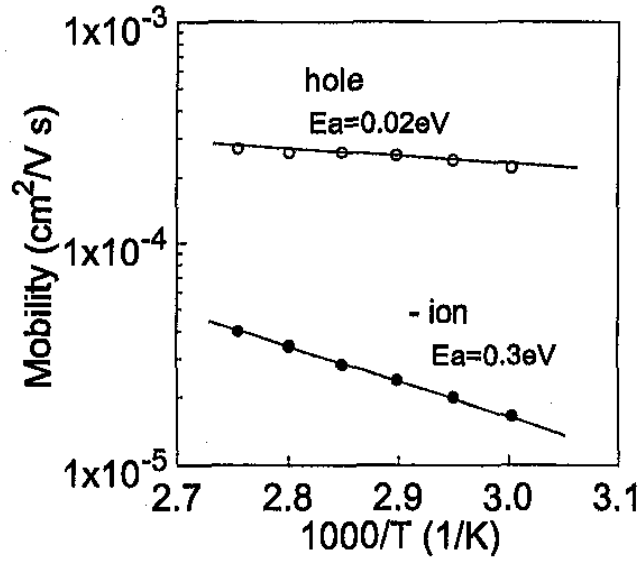

Fig. 4. Temperature dependences of the carrier mobility for hole (open circles) and negative ion (closed circles) in HHOTP in the discotic phase.

from traps. It should be mentioned that absorption coefficient $\alpha$ evaluated by this curve fitting method is consistent with the directly observed value estimated by comparing with incident and transmitted light intensities.

Utilizing the estimated transit time $t_{\tau}$, the carrier mobility $\mu$ was evaluated by the relation of $\mu=d^{2} / t_{\tau} V$, where $V$ is the applied voltage. Indeed, the transit time was proportioned to the reciprocal of applied field, $1 / E$, as shown in Fig. 3. The mobility evaluated from the slope of this dependence was around $2.6 \times 10^{-4} \mathrm{~cm}^{2} / \mathrm{Vs}$ at $80^{\circ} \mathrm{C}$ and temperature insensitive as shown in Fig. 4, which indicates that the migrating positive carriers are holes but not positive ions.

On the other hand, the mobility of the negative carri-

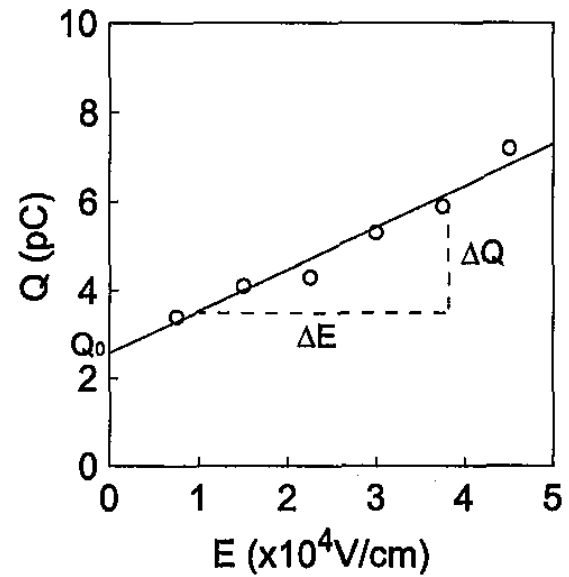

Fig. 6. Field strength dependence of the collected positive charge due to the hole migration in the discotic phase of HHOTP.

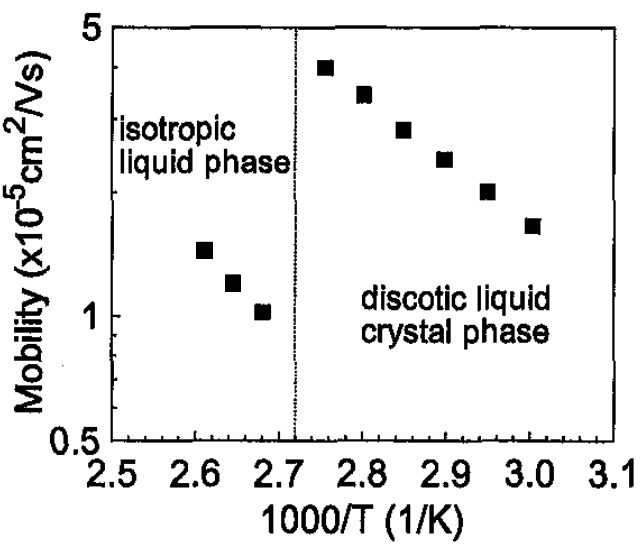

Fig, 5. Temperature dependence of the carrier mobility for negative ion in the isotropic and discotic of HHOTP.

ers is evaluated as $2.8 \times 10^{-5} \mathrm{~cm}^{2} / \mathrm{Vs}$ at $80^{\circ} \mathrm{C}$ and increases with increasing temperature with an activation energy of about $0.3 \mathrm{eV}$ as shown in Fig. 4. It is reasonable to interpret this negative carrier with a small mobility and large activation energy as ionic carrier, anion.

In the isotropic phase, the light induced current under positive bias decreases monotonously with time. On the other hand, under negative bias the photo-induced current exhibited similar wave form with that in the discotic phase, from which we can estimate the mobility. The mobility of negative carrier was $1.0 \times 10^{-5} \mathrm{~cm}^{2} / \mathrm{Vs}$ at $100^{\circ} \mathrm{C}$ and increased with increasing temperature with an activation energy of $0.4 \mathrm{eV}$. This negative carrier mobility in the isotropic phase was smaller than that in the discotic phase. This result suggests that the negative ionic carriers are migrating in HHOTP upon optical excitation under negative bias.

The drastic step wise drop of negative ion mobility was observed at the phase transition between discotic and isotropic phases with increasing temperature as shown in Fig. 5. This indicates that even for ionic carrier the migration is easier in the ordered state along columns. High ionic mobility in the ordered smectic phase compared with that in the disordered isotropic phase was also observed in the smectic liquid crystal [4].

By integrating photo-current signal with time, the collected charge can be evaluated.

The induced current signal in the crystal phase of HHOTP shows only long exponential decay for both 
positive and negative biases. Therefore we can not estimate the mobilities of both negative and positive carriers in the crystalline state. As evident in Fig. 6, the collected positive charge evaluated by integrating current signal due to hole migration in the discotic liquid crystal phase of HHOTP is proportional to the field strength. The slope-to-intercept value ( $\mathrm{s} /$ in), which is estimated from Fig. 6, is $3.1 \times 10^{-5} \mathrm{~cm} / \mathrm{V}$.

On the other hand, the escape probability $P$ of excited hole and electron pairs of separation distance $r$, under electric field $E$ at temperature $T$ is given by the following eq. (4) in the prediction of Onsager's theory [11],

$$
P=e^{-r_{c} / r}\left(1+\frac{e_{0}^{3}}{8 \pi \varepsilon_{0} \varepsilon_{r} k_{B}^{2} T^{2}} E\right) .
$$

Here, $\varepsilon_{\mathrm{r}}$ is relative dielectric constant, $k_{\mathrm{B}}$ is the Boltzmann constant, $r_{\mathrm{c}}$ is a critical distance. A critical distance $r_{\mathrm{c}}$ is defined by the condition that the Coulomb energy of attraction equals to the thermal energy of the medium. From eq. (4), the escape probability increases linearly with field strength as observed in the experiment and the slope-tointercept value, that is, the slope $d Q / d E$ in Fig. 6 divided by $Q_{0}$ is theoretically given by

$$
\frac{s l}{\text { in }}=\frac{e_{0}^{3}}{8 \pi \varepsilon_{0} \varepsilon_{r} k_{B}^{2} T^{2}}
$$

With $\varepsilon_{\mathrm{r}}=2.95$ and $T=343 \mathrm{~K}$, the theoretical slope-tointercept value is calculated to be $2.8 \times 10^{-5} \mathrm{~cm} / \mathrm{V}$. It should be stressed that there is a good agreement between experimental and theoretical values.

These facts mean that, in discotic liquid crystal, Onsager theory can be well applied as carrier generation process. That is, in Fig. 6, the linear portion is described by the influence of the electric field strength on the geminate recombination of hole/electron pairs which were generated by light absorption.

Besides, from the intercept at $E=0$ of the linear extrapolation of the photocurrent, the quantum yield for hole/electron pair generation by light absorption is obtained. The zero field quantum yield which was calculated by the intercept at $E=0$ and photon flux which was irradiated to HHOTP was approximately $0.05 \%$.

\section{Summary}

The present experimental study is summarized as follows.

(1) The mobilities of positive and negative carriers were estimated with curve fitting method by taking the initial spatial distribution of photo-generated charge carrier density in the discotic phase of HHOTP into consideration.

(2) In the discotic phase of HHOTP, temperature insensitive hole mobility as large as $2.6 \times 10^{-4} \mathrm{~cm}^{2} / \mathrm{Vs}$ was evaluated. On the other hand, the mobility for the negative carriers was evaluated to be $2.8 \times 10^{-5} \mathrm{~cm}^{2} /$ $\mathrm{Vs}$ at $80^{\circ} \mathrm{C}$ and increased with increasing temperature with an activation energy of $0.3 \mathrm{eV}$, from which the negative carrier was interpreted to be anions.

(3) Carrier (hole/electron pairs) generation process upon optical excitation can be explained in terms of Onsager model.

(4) Zero field quantum efficiency was evaluated to be $0.05 \%$ in the discotic phase.

\section{References}

[1] W. F. Schmidt and A. O. Allen: J. Chem. Phys. 50 (1969) 5037.

[2] R. M. Minday, L. O. Schmidt and H. T. Davis: J. Chem. Phys. 50 (1969) 1473.

[3] K. Yoshino, S. Hisamitsu and Y. Inuishi: J. Phys. Soc. Jpn. 32 (1972) 867.

[4] K. Yoshino, N. Tanaka and Y. Inuishi: Jpn. J. Appl. Phys. 15 (1976) 735.

[5] D. Adam, W. Rokhildt and D. Haarer: Jpn. J. Appl. Phys. 35 (1996) 1826.

[6] D. Adam, F. Closs, T. Frey, D. Funhoff, D. Haarer, H. Rigsdorf, P. Schuhmacher and K. Siemensmeyer: Phys. Rev. Lett. 70 (1993) 457.

[7] K. Yoshino, H. Nakayama, M. Ozaki, M. Onoda and M. Hamaguchi: Jpn. J. Appl. Phys. 36 (1997) 5183

[8] L. Y. Chiang, C. R. Safinya, N. A. Clark, K. S. Liang and A. N. Bloch: J. Chem. Soc. Chem. Commun. (1985) 695.

[9] C. Destrade, M. C. Mondon and J. Malthete: J. Phys. (Paris) 40 (1979) C3-17.

[10]K. Yoshino: IEEE Trans. Electr. Insul. 21 (1986) 999. [11]L. Onsager; Phys, Rev. 54 (1938) 554. 